\title{
Comparison of Speeds of Convergence in Riesz-Type Families of Summability Methods. II*
}

\author{
A. Šeletski and A. Tali \\ Institute of Mathematics and Natural Sciences, Tallinn University \\ Narva Road 25, 10120 Tallinn, Estonia \\ E-mail(corresp.): annatar@hot.ee, atali@tlu.ee
}

Received June 26, 2009; revised October 29, 2009; published online February 15, 2010

\begin{abstract}
Certain summability methods for functions and sequences are compared by their speeds of convergence. The authors are extending their results published in paper [9] for Riesz-type families $\left\{A_{\alpha}\right\}\left(\alpha>\alpha_{0}\right)$ of summability methods $A_{\alpha}$. Note that a typical Riesz-type family is the family formed by Riesz methods $A_{\alpha}=(R, \alpha), \alpha>0$. In [9] the comparative estimates for speeds of convergence for two methods $A_{\gamma}$ and $A_{\beta}$ in a Riesz-type family $\left\{A_{\alpha}\right\}$ were proved on the base of an inclusion theorem. In the present paper these estimates are improved by comparing speeds of three methods $A_{\gamma}$, $A_{\beta}$ and $A_{\delta}$ on the base of a Tauberian theorem. As a result, a Tauberian remainder theorem is proved. Numerical examples given in [9] are extended to the present paper as applications of the Tauberian remainder theorem proved here.
\end{abstract}

Keywords: speed of convergence, Tauberian remainder theorem, Riesz-type family of summability methods, Riesz methods, generalized integral Nörlund methods, Boreltype methods.

AMS Subject Classification: 40C10; 40E05; 40G05; 40G10.

\section{Introduction and Basic Notions}

We continue comparing speeds of convergence in Riesz-type families of summability methods started in paper [9]. In the mentioned paper any two methods in a Riesz-type family were compared by speed of convergence. In the present paper we improve our estimates comparing by speed of convergence any three methods in a Riesz-type family.

1.1. We begin our paper recalling the basic notions used in [9]. Let us consider functions $x=x(u)$ defined for $u \geq 0$, bounded and Lebesgue-measurable on every finite interval $\left[0, u_{0}\right]$. Let us denote the set of all such functions by $X$.

\footnotetext{
* This research was supported by the Estonian Science Foundation (grants No. 7033 and No. 7319).
} 
Suppose that $A$ is a transformation of functions $x=x(u)$ (or, in particular, of sequences $\left.x=\left(x_{n}\right)\right)$ into functions $A x=y=y(u) \in X$. If the limit $\lim _{u \rightarrow \infty} y(u)=s$ exists then we say that $x=x(u)$ is convergent to $s$ with respect to the summability method $A$, and write $x(u) \rightarrow s(A)$. If $y=y(u)$ is bounded then we say that $x$ is bounded with respect to $A$, and write $x(u)=O(A)$. We denote by $\omega A$ the set of all these functions $x$, where the transformation $A$ is applied, and by $c A$ and $m A$ the set of all functions $x$ which are convergent and bounded with respect to the method $A$, respectively. The method $A$ is said to be regular if $\lim _{u \rightarrow \infty} x(u)=s$ implies $\lim _{u \rightarrow \infty} y(u)=s$ whenever $x \in X$. Further we use the notation $c_{0}$ for the set of all functions $x \in X$ having $\lim _{u \rightarrow \infty} x(u)=0$.

One of the most common summability method for functions $x$ is an integral method $A$ is defined with the help of transformation

$$
y(u)=\int_{0}^{\infty} a(u, v) x(v) d v,
$$

where $a(u, v)$ is a certain function of two variables $u \geq 0$ and $v \geq 0$. We say also that the integral method $A$ is defined by the function $a(u, v)$. An example of an integral summability method is the generalized integral Nörlund method $(N, P(u), Q(u))$ defined with the help of transformation

$$
y(u)=\frac{1}{R(u)} \int_{0}^{u} P(u-v) Q(v) x(v) d v \quad(u>0),
$$

where $P=P(u)$ and $Q=Q(u)$ are non-negative functions from $X$ such that

$$
R(u)=\int_{0}^{u} P(u-v) Q(v) d v \neq 0 \quad \text { for } u>0 .
$$

In particular, if $Q(u)=1$ and $P(u)=u^{\alpha-1}$ for $u>0$ and $\alpha>0$, we get the Riesz method $(R, \alpha)$.

For sequences $x=\left(x_{n}\right)$ we focus ourselves on certain semi-continuous summability methods $A$ defined by transformations

$$
y(u)=\sum_{n=0}^{\infty} a_{n}(u) x_{n} \quad(u \geq 0)
$$

where $a_{n}(u)(n=0,1, \ldots)$ are some functions from $X$. An example of a semicontinuous method is the Borel method $B$ defined by the transformation

$$
y(u)=\frac{1}{e^{u}} \sum_{n=0}^{\infty} \frac{u^{n}}{n !} x_{n} .
$$

1.2. One of the basic notions in this paper is the "speed of convergence". We use here definitions based on the definitions for sequences (see [4] and [5]) and extended for functions in [8] and [12]. Let $\lambda=\lambda(u)$ be a positive function from $X$ such that $\lambda(u) \rightarrow \infty$ as $u \rightarrow \infty$. It is said that a function $x=x(u)$ is convergent to $s$ with speed $\lambda$ (shortly: $\lambda$-convergent) if the finite 
limit $\lim _{u \rightarrow \infty} \lambda(u)[x(u)-s]$ exists. If $\lambda(u)[x(u)-s]=O(1)$ as $u \rightarrow \infty$, then $x$ is said to be $\lambda$-bounded.

We use the notations $c^{\lambda}$ and $m^{\lambda}$ for the sets of all $\lambda$-convergent and $\lambda$ bounded functions $x$, respectively. It is said that $x$ is convergent or bounded with speed $\lambda$ with respect to the summability method $A$ if $A x \in c^{\lambda}$ or $A x \in m^{\lambda}$, respectively.

1.3. The main subject of the paper is a Riesz-type family of summability methods $([8,13])$. Let $\left\{A_{\alpha}\right\}$ be a family of summability methods $A_{\alpha}$ where ${ }^{1} \alpha_{(-)}^{>} \alpha_{1}$ and which are defined by transformations of functions $x=x(u) \in$ $\omega A_{\alpha} \subset X$ into $A_{\alpha} x=y_{\alpha}=y_{\alpha}(u) \in X$. Suppose that for any $\beta>\gamma_{(-)}^{>} \alpha_{1}$ we have

$$
\omega A_{\gamma} \subset \omega A_{\beta} .
$$

Definition 1. ([8], Definition 1; [13], Definition 2) A family $\left\{A_{\alpha}\right\}\left(\alpha_{(-)}^{>} \alpha_{1}\right)$ is said to be a Riesz-type family if for every $\beta>\gamma_{(-)}^{>} \alpha_{1}$ the relation (1.2) holds and the methods $A_{\gamma}$ and $A_{\beta}$ are connected through

$$
\begin{aligned}
& y_{\beta}(u)=\frac{M_{\gamma, \beta}}{r_{\beta}(u)} \int_{0}^{u}(u-v)^{\beta-\gamma-1} r_{\gamma}(v) y_{\gamma}(v) d v \quad(u>0), \\
& r_{\beta}(u)=M_{\gamma, \beta} \int_{0}^{u}(u-v)^{\beta-\gamma-1} r_{\gamma}(v) d v \quad(u>0),
\end{aligned}
$$

where $r_{\gamma}=r_{\gamma}(u)$ and $r_{\beta}=r_{\beta}(u)$ are some positive functions from $X$ and $M_{\gamma, \beta}$ is a constant depending on $\gamma$ and $\beta$.

Example 1. Let $\left\{A_{\alpha}\right\}$ be the family of generalized Nörlund methods $A_{\alpha}=$ $\left(N, p_{\alpha}(u), q(u)\right)\left(\alpha>\alpha_{0}\right)$ defined by positive functions $p=p(u) \in X$ and $q=q(u) \in X$ and a number $\alpha_{0}$ such that

$$
r_{\alpha}(u)=\int_{0}^{u} p_{\alpha}(u-v) q(v) d v>0 \quad\left(u>0, \alpha>\alpha_{0}\right),
$$

where $p_{\alpha}(u)=\int_{0}^{u}(u-v)^{\alpha-1} p(v) d v$. It is known that relations (1.3) together with (1.4) and (1.6) hold here for any $\beta>\gamma>\alpha_{0}$ (see [14]), and thus this family is a Riesz-type family.

Example 2. Consider the Borel-type methods $A_{\alpha}=\left(B, \alpha, q_{n}\right)$ (see [13]). Let $\left(q_{n}\right)$ be a non-negative sequence such that the power series $\sum q_{n} u^{n}$ has the radius of convergence $R=\infty$ and $q_{n}>0$ at least for one $n \in \mathbf{N}$. Denote

$$
r_{\alpha}(u)=\sum_{n=1}^{\infty} \frac{n ! q_{n} u^{n+\alpha-1}}{\Gamma(n+\alpha)}
$$

and define the methods $\left(B, \alpha, q_{n}\right)(\alpha>-1 / 2)$ for converging sequences $x=\left(x_{n}\right)$ with the help of transformation

$$
y_{\alpha}(u)=\frac{1}{r_{\alpha}(u)} \sum_{n=1}^{\infty} \frac{n ! q_{n} u^{n+\alpha-1}}{\Gamma(n+\alpha)} x_{n} \quad(u>0) .
$$

\footnotetext{
1 The notation $\alpha_{(-)}^{>} \alpha_{1}$ means that we consider parameter values $\alpha>\alpha_{1}$ or $\alpha \geq \alpha_{1}$ with some fixed number $\alpha_{1}$.
} 
The methods $A_{\alpha}=\left(B, \alpha, q_{n}\right)$ satisfy relations (1.3) and (1.4) with $r_{\alpha}(u)$ defined by (1.5) and $M_{\gamma, \beta}=1 / \Gamma(\beta-\gamma)$ (see [13]) and form therefore a Riesz-type family. In particular, if $q_{n}=\frac{1}{n !}$ we get the Borel-type methods $(B, \alpha)=$ $(B, \alpha, 1 / n !)$ (see $[1,2])$. If, in addition, $\alpha=1$, we have the Borel method $B=(B, 1)$.

Example 3. Consider the family of generalized Nörlund methods $A_{\alpha}=\left(N, u^{\alpha-1}\right.$, $q(u))$ where $\alpha>0$ and $q=q(u)$ is a positive function from $X$. These methods are defined by transformation of $x$ into $A_{\alpha} x=y_{\alpha}(u)$ with

$$
y_{\alpha}(u)=\frac{1}{r_{\alpha}(u)} \int_{0}^{u}(u-v)^{\alpha-1} q(v) x(v) d v \quad(u>0),
$$

where $r_{\alpha}=r_{\alpha}(u)=\int_{0}^{u}(u-v)^{\alpha-1} q(v) d v$. This family satisfies relations (1.3) and (1.4) with

$$
M_{\gamma, \beta}=\frac{\Gamma(\beta)}{\Gamma(\gamma) \Gamma(\beta-\gamma)}
$$

(see [9], Example 1) and therefore it is a Riesz-type family. In particular, if $q(u)=1(u \geq 0)$ we have Riesz methods $\left(N, u^{\alpha-1}, 1\right)=(R, \alpha)$.

\section{Preliminary Results}

We need some results proved in [9].

2.1. Speeds of convergence of any two methods in a Riesz-type family were compared in [9] on the base of an inclusion theorem which will be formulated as the following proposition.

Proposition 1. Let $\left\{A_{\alpha}\right\}\left(\alpha_{(-)}^{>} \alpha_{1}\right)$ be a Riesz-type family. Then we have for functions $x=x(u)$ and numbers $s$ and $\beta>\gamma_{(-)}^{>} \alpha_{1}$ that

i) $x(u)=O\left(A_{\gamma}\right) \Longrightarrow x(u)=O\left(A_{\beta}\right)$, ii) $x(u) \rightarrow s\left(A_{\gamma}\right) \Longrightarrow x(u) \rightarrow s\left(A_{\beta}\right)$, provided in case ii) that $\lim _{u \rightarrow \infty} \int_{0}^{u} r_{\alpha_{1}}(v) d v=\infty$ is satisfied if $\gamma=\alpha_{1}$ is included.

The next theorem (see [9], Theorem 1) describes how the speed of convergence changes if we go from one summability method in the family to a stronger one.

Theorem A. Let $\left\{A_{\alpha}\right\}\left(\alpha>\alpha_{0}\right)$ be a Riesz-type family. Let some positive function $\lambda=\lambda(u) \rightarrow \infty$ (as $u \rightarrow \infty)$ from $X$ and some number $\gamma>\alpha_{0}$ such that $\frac{r_{\gamma}(u)}{\lambda(u)} \in X$ be given.

i) Then we have for functions $x=x(u)$ and numbers $s$ and $\beta>\gamma$ that

$$
\lambda(u)\left[y_{\gamma}(u)-s\right]=O(1) \Longrightarrow \lambda_{\beta}(u)\left[y_{\beta}(u)-s\right]=O(1),
$$

where the speeds are related through the formulas

$$
\lambda_{\beta}(u)=\frac{r_{\beta}(u)}{b_{\beta}(u)}, b_{\beta}(u)=M_{\gamma, \beta} \int_{0}^{u}(u-v)^{\beta-\gamma-1} b_{\gamma}(v) d v, b_{\gamma}(u)=\frac{r_{\gamma}(u)}{\lambda(u)} .
$$


ii) Moreover, we have that

$$
\lambda(u)\left[y_{\gamma}(u)-s\right] \rightarrow t \Longrightarrow \lambda_{\beta}(u)\left[y_{\beta}(u)-s\right] \rightarrow t,
$$

provided that

$$
\lim _{u \rightarrow \infty} \int_{0}^{u} b_{\gamma}(v) d v=\infty
$$

Under restriction $(2.4)$ the condition $\lambda(u) \rightarrow \infty$ implies $\lambda_{\beta}(u) \rightarrow \infty$ in Theorem A (see [9], Remark 2). We note also that Theorem A can be considered as a generalization of case A) of Theorem 1 from [12], which was proved for matrix case. Certain evaluations for speed of convergence for Riesz and Nörlund matrix methods in Banach spaces were proved in recent papers [6] and [7].

2.2. The speeds $\lambda=\lambda(u)$ and $\lambda_{\beta}=\lambda_{\beta}(u)$ defined in Theorem A can be compared by the inequalities.

Let $a=a(u)$ and $b=b(u)$ be two positive functions from $X$. If there exist positive numbers $c_{1}, c_{2}$ and $u_{0}$ such that the condition

$$
c_{1} b(u) \leq a(u) \leq c_{2} b(u)
$$

holds for every $u>u_{0}$, we write $a(u) \approx b(u)$. If $b=b(u)$ is nondecreasing and condition (2.5) is satisfied with some positive $c_{1}$ and $c_{2}$ for any $u>0$, then we say that $a=a(u)$ is almost nondecreasing.

The following proposition is proved in [9] (see [9], Propositions 2 and 3).

Proposition 2. Let a Riesz-type family $\left\{A_{\alpha}\right\}\left(\alpha>\alpha_{0}\right)$ and a positive function $\lambda=\lambda(u) \in X$ be given. Fix some $\gamma>\alpha_{0}$ and suppose that $\lambda_{\beta}=\lambda_{\beta}(u)$ $\left(\beta>\gamma>\alpha_{0}\right)$ is defined through (2.2). Then for $\beta>\gamma>\alpha_{0}$ we have:

i) $\lambda_{\beta}(u) \leq L \lambda(u)(u>0)$ provided that $\lambda=\lambda(u)$ is almost nondecreasing,

ii) $\lambda_{\beta}(u) \geq \frac{K r_{\beta}(u)}{r_{\gamma}(u) u^{\beta-\gamma}} \lambda(u) \quad(u>0)$ provided that $b_{\gamma}(u)=r_{\gamma}(u) / \lambda(u)$ is almost nondecreasing, where $L$ and $K$ are some positive constants independent from $u$.

Previous result state that switching to a stronger method, the speed of convergence can not be improved but also it cannot become too much worse. This is consistent with results known for matrix methods (see e.g. [4, 6, 12]).

\section{Main Results. A Tauberian Remainder Theorem}

First we prove a convexity theorem.

Theorem 1. Let $\left\{A_{\alpha}\right\}\left(\alpha_{(-)}^{>} \alpha_{1}\right)$ be a Riesz-type family satisfying the condition

$$
r_{\beta}(u) / r_{\alpha}(u) \approx u^{\beta-\alpha} \quad(u>0)
$$

for all $\beta>\alpha>\alpha_{1}$. Then we have for functions $x=x(u)$ and numbers $s$ and $\beta>\delta>\gamma_{(-)}^{>} \alpha_{1}$ that

$$
x(u)=O\left(A_{\gamma}\right), \quad x(u) \rightarrow s\left(A_{\beta}\right) \Longrightarrow x(u) \rightarrow s\left(A_{\delta}\right) .
$$


Proof. Suppose first that $\gamma>\alpha_{1}$. Without a loss of generality we may take $\beta=\gamma+1$ and $s=0$. Suppose that

$$
y_{\gamma+1}(u) \rightarrow 0 \text { as } u \rightarrow \infty, \quad y_{\gamma}(u)=O(1)
$$

for a function $x=x(u)$ and some value $\gamma$ of the parameter, and show that

$$
y_{\delta}(u) \rightarrow 0 \text { as } u \rightarrow \infty
$$

for any $\delta$ such that $\gamma<\delta<\gamma+1$. By relation (1.3) we have that

$$
y_{\delta}(u)=\frac{M_{\gamma, \delta}}{r_{\delta}(u)} \int_{0}^{u}(u-v)^{\delta-\gamma-1} r_{\gamma}(v) y_{\gamma}(v) d v \quad(u>0) .
$$

Choose some $\theta \in(1 / 2 ; 1)$ and divide $y_{\delta}(u)$ into two parts:

$$
\begin{aligned}
y_{\delta}(u) & =\frac{M_{\gamma, \delta}}{r_{\delta}(u)} \int_{0}^{\theta u}(u-v)^{\delta-\gamma-1} r_{\gamma}(v) y_{\gamma}(v) d v \\
& +\frac{M_{\gamma, \delta}}{r_{\delta}(u)} \int_{\theta u}^{u}(u-v)^{\delta-\gamma-1} r_{\gamma}(v) y_{\gamma}(v) d v=I_{1}(u, \theta)+I_{2}(u, \theta) .
\end{aligned}
$$

Thus we have the equality $y_{\delta}(u)=I_{1}(u, \theta)+I_{2}(u, \theta)$. Note that $I_{1}(u, \theta)$ and $I_{2}(u, \theta)$ depend also on $\gamma, \delta$. Integrating by parts, we get for $I_{1}(u, \theta)$ the following form:

$$
\begin{aligned}
& I_{1}(u, \theta)=\left.\frac{M_{\gamma, \delta}}{r_{\delta}(u)}\left((u-v)^{\delta-\gamma-1} \int_{0}^{v} r_{\gamma}(t) y_{\gamma}(t) d t\right)\right|_{0} ^{\theta u} \\
& +\frac{M_{\gamma, \delta}}{r_{\delta}(u)} \int_{0}^{\theta u}\left[(\delta-\gamma-1)(u-v)^{\delta-\gamma-2} \int_{0}^{v} r_{\gamma}(t) y_{\gamma}(t) d t\right] d v=I_{1}^{\prime}(u, \theta)+I_{1}^{\prime \prime}(u, \theta),
\end{aligned}
$$

where

$$
\begin{aligned}
I_{1}^{\prime}(u, \theta) & =\left.\frac{M_{\gamma, \delta}}{r_{\delta}(u)}\left((u-v)^{\delta-\gamma-1} \int_{0}^{v} r_{\gamma}(t) y_{\gamma}(t) d t\right)\right|_{0} ^{\theta u}=\frac{M_{\gamma, \delta}}{r_{\delta}(u)}(u-\theta u)^{\delta-\gamma-1} \\
& \times \int_{0}^{\theta u} r_{\gamma}(t) y_{\gamma}(t) d t=\frac{M_{\gamma, \delta}}{M_{\gamma, \gamma+1}} \frac{(u-\theta u)^{\delta-\gamma-1}}{r_{\delta}(u)} r_{\gamma+1}(\theta u) y_{\gamma+1}(\theta u)
\end{aligned}
$$

and

$$
\begin{aligned}
I_{1}^{\prime \prime}(u, \theta) & =\frac{M_{\gamma, \delta}}{r_{\delta}(u)} \int_{0}^{\theta u}\left[(\delta-\gamma-1)(u-v)^{\delta-\gamma-2} \int_{0}^{v} r_{\gamma}(t) y_{\gamma}(t) d t\right] d v \\
& =\frac{M_{\gamma, \delta}}{M_{\gamma, \gamma+1}} \frac{1}{r_{\delta}(u)} \int_{0}^{\theta u}(\delta-\gamma-1)(u-v)^{\delta-\gamma-2} r_{\gamma+1}(v) y_{\gamma+1}(v) d v .
\end{aligned}
$$

Using conditions (3.1) and (3.3) we get

$$
\begin{aligned}
I_{1}^{\prime}(u, \theta) & =O(1) \frac{(u-\theta u)^{\delta-\gamma-1}}{r_{\delta}(u)} r_{\gamma+1}(u) y_{\gamma+1}(u)=O(1) u^{\gamma+1-\delta} u^{\delta-\gamma-1} \\
& \times(1-\theta)^{\delta-\gamma-1} y_{\gamma+1}(u)=o(1)(1-\theta)^{\delta-\gamma-1}=o_{\theta}(1) \text { as } u \rightarrow \infty .
\end{aligned}
$$


Thus we have $I_{1}^{\prime}(u, \theta)=o_{\theta}(1)$ as $u \rightarrow \infty$. Let us show that also $I_{1}^{\prime \prime}(u, \theta)=o_{\theta}(1)$ as $u \rightarrow \infty$. Denoting

$$
c_{\gamma, \delta}^{\prime}(u, v)= \begin{cases}\frac{1}{r_{\delta}(u)}(u-v)^{\delta-\gamma-2} r_{\gamma+1}(v), & \text { if } 0 \leq v \leq \theta u, \\ 0, & \text { if } v>\theta u,\end{cases}
$$

we will show that the integral transformation defined by $c_{\gamma, \delta}^{\prime}(u, v)$ is a $c_{0} \rightarrow c_{0}$ type transformation. We use Theorem 6 from [3] which gives the sufficient conditions for the regularity of integral methods. Let us prove first that

$$
\int_{0}^{v_{0}} c_{\gamma, \delta}^{\prime}(u, v) d v=o_{\theta}(1) \text { as } u \rightarrow \infty
$$

assuming that $v_{0}$ is a fixed positive number and $v<v_{0}<\theta u$. We get:

$$
\begin{aligned}
& \int_{0}^{v_{0}} c_{\gamma, \delta}^{\prime}(u, v) d v=\frac{1}{r_{\delta}(u)} \int_{0}^{v_{0}}(u-v)^{\delta-\gamma-2} r_{\gamma+1}(v) d v \leq \frac{r_{\gamma+1}(u)}{r_{\delta}(u)} \\
& \quad \times \int_{0}^{v_{0}}(u-v)^{\delta-\gamma-2} d v=\left.O(1) u^{\gamma+1-\delta}(u-v)^{\delta-\gamma-1}\right|_{0} ^{v_{0}} \\
& \quad=O(1)\left[u^{\gamma+1-\delta}\left(u-v_{0}\right)^{\delta-\gamma-1}-1\right] \\
& \quad=O(1)\left[\left(1-\frac{v_{0}}{u}\right)^{\delta-\gamma-1}-1\right]=o_{\theta}(1) \text { as } u \rightarrow \infty
\end{aligned}
$$

Following Theorem 6 from [3] it remains to show that the condition

$$
\int_{0}^{\theta u} c_{\gamma, \delta}^{\prime}(u, v) d v=O_{\theta}(1) \quad(u>0)
$$

is also fulfilled. With the help of (3.1) we get:

$$
\begin{aligned}
\int_{0}^{\theta u} \frac{r_{\gamma+1}(v)}{r_{\delta}(u)}(u-v)^{\delta-\gamma-2} d v & \leq \frac{r_{\gamma+1}(u)}{r_{\delta}(u)} \int_{0}^{\theta u}(u-v)^{\delta-\gamma-2} d v \\
& =O(1) u^{\gamma+1-\delta}(u-\theta u)^{\delta-\gamma-1}=O_{\theta}(1) .
\end{aligned}
$$

Thus we have shown that the integral transformation defined by $c_{\gamma, \delta}^{\prime}(u, v)$ is of type $c_{0} \rightarrow c_{0}$ for every $\theta \in(1 / 2 ; 1)$, and therefore condition $I_{1}^{\prime \prime}(u, \theta)=o_{\theta}(1)$ is satisfied. By the obtained relations we have that

$$
I_{1}(u, \theta)=I_{1}^{\prime}(u, \theta)+I_{1}^{\prime \prime}(u, \theta)=o_{\theta}(1) \quad \text { as } u \rightarrow \infty .
$$

Next we evaluate the quantity $I_{2}(u, \theta)$ using relations (3.1) and (3.3):

$$
\begin{aligned}
& I_{2}(u, \theta)=O(1) \int_{\theta u}^{u}(u-v)^{\delta-\gamma-1} \frac{r_{\gamma+1}(v)}{v r_{\delta}(u)} d v=O(1) \frac{r_{\gamma+1}(u)}{r_{\delta}(u) \theta u} \\
& \quad \times \int_{\theta u}^{u}(u-v)^{\delta-\gamma-1} d v=\left.O(1) u^{\gamma-\delta}(u-v)^{\delta-\gamma}\right|_{u} ^{\theta u}=O(1)(1-\theta)^{\delta-\gamma} .
\end{aligned}
$$

So we have the estimate

$$
I_{2}(u, \theta)=O(1)(1-\theta)^{\delta-\gamma} .
$$


Now we are able to complete our proof showing that (3.4) is true for every $\gamma<\delta<\gamma+1$. We choose $\varepsilon>0$ and afterwards $\theta_{\varepsilon} \in(1 / 2,1)$ so, that

$$
I_{2}\left(u, \theta_{\varepsilon}\right)=O(1)\left(1-\theta_{\varepsilon}\right)^{\delta-\gamma}<\frac{\varepsilon}{2} \text { for any } u>0
$$

(see (3.7)). Next we choose $U=U_{\theta_{\varepsilon}}$ so, that $\left|I_{1}\left(u, \theta_{\varepsilon}\right)\right|<\varepsilon / 2$ for all $u>U$ (see (3.6)). It follows from (3.5) that $\left|y_{\delta}(u)\right|<\varepsilon$ when $u>U$, i.e., (3.4) holds. Thus we have shown that implication (3.2) is true for all $\beta>\delta>\gamma>\alpha_{1}$.

If $\gamma=\alpha_{1}$, then we choose some $\gamma<\gamma_{1}<\delta$ and get that $x(u)=O\left(A_{\gamma}\right)$ implies $x(u)=O\left(A_{\gamma_{1}}\right)$. To finish the proof, it remains to apply implication (3.2), already proved, with $\gamma_{1}$ instead of $\gamma$.

Note that Theorem 1 was formulated (but not proved) in [13] as Proposition 4 with a hint on analogy with matrix case (see $[10,11])$. The following Tauberian remainder theorem extends Theorem A.

Theorem 2. Let $\left\{A_{\alpha}\right\}\left(\alpha>\alpha_{0}\right)$ be a Riesz-type family. Let some positive function $\lambda=\lambda(u) \rightarrow \infty($ as $u \rightarrow \infty)$ from $X$ and some number $\gamma>\alpha_{0}$ such that $r_{\gamma}(u) / \lambda(u) \in X$ be given. Suppose that $b_{\beta}(u)$ and $\lambda_{\beta}(u)$ are defined through (2.2). Suppose also that the following condition

$$
b_{\beta}(u) / b_{\alpha}(u) \approx u^{\beta-\alpha} \quad(u>0)
$$

is satisfied for any $\beta>\alpha>\gamma$. Then we have for functions $x=x(u)$ and numbers $s$ and $\beta>\delta>\gamma$ that

$$
\lambda(u)\left[y_{\gamma}(u)-s\right]=O(1), \lambda_{\beta}(u)\left[y_{\beta}(u)-s\right] \rightarrow t \Longrightarrow \lambda_{\delta}(u)\left[y_{\delta}(u)-s\right] \rightarrow t .
$$

Proof. We set $\alpha_{1}=\gamma$ and construct another family $\left\{B_{\alpha}\right\}(\alpha \geq \gamma)$ on the base of relations (2.2). Namely, we define the methods $B_{\alpha}$ by the transformations of a function $y=y(u) \in X$ into $\eta_{\alpha}=\eta_{\alpha}(u)$ with

$$
\eta_{\alpha}(u)=\frac{M_{\gamma, \alpha}}{b_{\alpha}(u)} \int_{0}^{u}(u-v)^{\beta-\gamma-1} b_{\gamma}(v) y(v) d v \quad(\alpha>\gamma)
$$

and $\eta_{\gamma}(u)=y(u)$, i.e., $B_{\gamma}=I$. The family $\left\{B_{\alpha}\right\}(\alpha \geq \gamma)$ is a Riesz-type family (see Example 3) satisfying the presumptions of Theorem 1. Let us apply methods $B_{\alpha}$ to $y=\lambda(u)\left[y_{\gamma}(u)-s\right]$ and realize that $B_{\alpha} y=\eta_{\alpha}(u)=$ $\lambda_{\alpha}(u)\left[y_{\alpha}(u)-s\right]$ for any $\alpha>\gamma$. Thus, implication (3.9) holds by Theorem 1 for any $\beta>\delta>\gamma$ as (3.2) in the form

$$
y(u)=O\left(B_{\gamma}\right), \quad y(u) \rightarrow t\left(B_{\beta}\right) \Longrightarrow y(u) \rightarrow t\left(B_{\delta}\right) .
$$

An analogous Tauberian remainder theorem for "matrix case" was proved in [12] as Theorem 2. Some Tauberian remainder theorems for Nörlund and Riesz matrix methods in Banach spaces were proved recently in [6] and [7]. Some estimates for speeds in a Riesz-type family (weaker than here) can be found also in [8]. 


\section{Examples on Comparison of Speeds of Convergence}

Here we give some numerical examples on application of Theorem 2 for comparison of speeds of convergence in special Riesz-type families. More precisely, we extend Examples 5, 7 and 9 from [9], where Theorem A was applied. In mentioned examples comparative evaluations (2.1) and (2.3) for speeds of any two methods $A_{\gamma}$ and $A_{\beta}$ in Riesz-type families $\left\{A_{\alpha}\right\}$ are presented. Here we improve these results, comparing any three methods $A_{\gamma}, A_{\beta}$ and $A_{\delta}$ with the help of implication (3.9).

Example 4. We consider the Riesz methods $A_{\alpha}=(R, \alpha)(\alpha>0)$. Choose the speed of convergence $\lambda(u)=(u+1)^{\rho}(\rho>0)$ and fix some number $\gamma>0$. Suppose that $x=x(u)$ is a function having a given speed of convergence $\lambda(u)$ with respect to the method $A_{\gamma}=(R, \gamma)$ and define with the help of formulas (2.2) the function $b_{\beta}(u)$ and afterwards the speed of convergence $\lambda_{\beta}(u)$ of $x=x(u)$ with respect to the methods $A_{\beta}=(R, \beta)$ for $\beta>\gamma$. In Example 5 in [9] the following estimates for $b_{\beta}(u)$ and $\lambda_{\beta}(u)$ were proved for any $\beta>\gamma$ if $u \rightarrow \infty$ :

$$
\begin{aligned}
& b_{\beta}(u) \sim M_{\gamma, \beta} B(\beta-\gamma, \gamma-\rho+1) u^{\beta-\rho} / \gamma, \quad \text { if } \rho<\gamma+1, \\
& b_{\beta}(u) \approx \begin{cases}u^{\beta-\gamma-1} \log u, & \text { if } \rho=\gamma+1, \\
u^{\beta-\gamma-1}, & \text { if } \rho>\gamma+1,\end{cases} \\
& \lambda_{\beta}(u) \sim \frac{\Gamma(\gamma+1) \Gamma(\beta-\rho+1)}{\Gamma(\beta+1) \Gamma(\gamma-\rho+1)} u^{\rho} \sim \frac{\Gamma(\gamma+1) \Gamma(\beta-\rho+1)}{\Gamma(\beta+1) \Gamma(\gamma-\rho+1)} \lambda(u), \\
& \text { if } \rho<\gamma+1 \text {, } \\
& \lambda_{\beta}(u) \approx \begin{cases}u^{\rho} / \log u \sim \lambda(u) / \log u, & \text { if } \rho=\gamma+1, \\
u^{\rho} u^{\gamma-\rho+1} \sim \lambda(u) u^{\gamma-\rho+1}, & \text { if } \rho>\gamma+1 .\end{cases}
\end{aligned}
$$

Estimates (4.1)-(4.2) show that condition (3.8) is satisfied for all $\beta>\alpha>\gamma$. Thus Theorem 2 applies, and implication (3.9) is true for any $\beta>\delta>\gamma$ where speeds $\lambda_{\beta}$ and $\lambda_{\delta}$ obey evaluates (4.3) and (4.4).

Example 5. Let us consider the Borel-type methods $A_{\alpha}=(B, \alpha, 1 / n !)=(B, \alpha)$ $(\alpha>-1 / 2)$. Suppose that $\lambda(u)=(u+1)^{\rho} e^{u}$, fix some $\gamma>-1 / 2$ and find $\lambda_{\beta}(u)$ for $\beta>\gamma$ through (2.2) again. In Example 7 in [9] for $\beta>\gamma$ the following estimates were proved:

$$
\begin{aligned}
& b_{\beta}(u) \approx \begin{cases}u^{\beta-\gamma-1}, & \text { if } \rho>1, \\
u^{\beta-\gamma-1} \log u, & \text { if } \rho=1, \\
u^{\beta-\gamma-\rho}, & \text { if } \rho<1,\end{cases} \\
& \lambda_{\beta}(u) \approx \begin{cases}\frac{e^{u}}{u^{\beta-\gamma-1}} \sim \frac{\lambda(u)}{u^{\beta-\gamma+\rho-1},} & \text { if } \rho>1, \\
\frac{e^{u}}{u^{\beta-\gamma-1} \log u} \sim \frac{\lambda(u)}{u^{\beta-\gamma} \log u}, & \text { if } \rho=1, \\
\frac{e^{u}}{u^{\beta-\gamma-\rho}} \sim \frac{\lambda(u)}{u^{\beta-\gamma}}, & \text { if } \rho<1 .\end{cases}
\end{aligned}
$$


Condition (3.8) is satisfied for all $\beta>\alpha>\gamma$ by relations (4.5). Therefore, Theorem 2 applies again, and implication (3.9) is true for any $\beta>\delta>\gamma$ where speeds $\lambda_{\beta}$ and $\lambda_{\delta}$ obey evaluates (4.6).

Example 6. Suppose that $A_{\alpha}=\left(N, u^{\alpha-1}, e^{u^{\varphi}}\right)(\alpha>0)$ where $0<\varphi<1$ is some fixed number. Suppose that $\lambda(u)=e^{u^{\varphi}}$. It was shown in Example 9 in [9] that $b_{\beta}(u) \approx u^{\beta-\gamma+(1-\varphi) \gamma}$ and $\lambda_{\beta}(u) \approx e^{u^{\varphi}} u^{\varphi(\gamma-\beta)}=u^{\varphi(\gamma-\beta)} \lambda(u)$ for $\beta>\gamma$. We see that (3.8) is satisfied for any $\beta>\alpha>\gamma$. Therefore, implication (3.9) is true for any $\beta>\delta>\gamma$ by Theorem 2 .

\section{References}

[1] D. Borwein. On Borel-type methods of summability. Mathematica, 5:128-133, 1958.

[2] D. Borwein and B. L. R. Shawyer. On Borel-methods. Tôhoku Math. J., 18:283298, 1966. Doi: $10.2748 / \mathrm{tmj} / 1178243418$.

[3] G.H. Hardy. Divergent series. Oxford Press, 1949.

[4] G. Kangro. On Bohr-Hardy-type multipliers of convergence for a given speed I. Proc. Estonian Acad. Sci. Phys. Math., 18:137-145, 1969. (in Russian)

[5] G. Kangro. Summability factors for the series $\lambda$-bounded by the methods of Riesz and Cesàro. Tartu Ülik. Toimetised, 277:136-154, 1971. (in Russian)

[6] O. Meronen and I. Tammeraid. Generalized Nörlund method and convergence acceleration. Math. Model. Anal., 12:195-204, 2007.

Doi:10.3846/1392-6292.2007.12.195-204.

[7] O. Meronen and I. Tammeraid. Generalized linear methods and gap Tauberian remainder theorems. Math. Model. Anal., 13:223-232, 2008. Doi:10.3846/1392-6292.2008.13.223-232.

[8] V. Pavlova and A. Tali. On the convexity theorem of M. Riesz. Proc. Estonian Acad. Sci. Phys. Math., 51:18-34, 2002.

[9] A. Šeletski and A. Tali. Comparison of speeds of convergence in Riesz-type families of summability methods. Proc. Estonian Acad. Sci., 57:70-80, 2008. Doi:10.3176/proc.2008.2.02.

[10] R. Sinha. Convexity theorem for $(N, p, q)$ summability. Kyungpook Math. J., 13:37-40, 1973.

[11] U. Stadtmüller and A. Tali. On some families of certain Nörlund methods and power series methods. J. Math. Anal. Appl., 238:44-66, 1999. Doi:10.1006/jmaa.1999.6503.

[12] U. Stadtmüller and A. Tali. Comparison of certain summability methods by speeds of convergence. Analysis Mathematica, 29:227-242, 2003. Doi:10.1023/A:1025419305735.

[13] U. Stadtmüller and A. Tali. Strong summability in certain families of summability methods. Acta Sci. Math. (Szeged), 70:639-657, 2004.

[14] A. Tali. Zero-convex families of summability methods. Tartu Ülik. Toimetised, 504:48-57, 1981. (in Russian) 